\title{
Utility of endobronchial ultrasound guided trans-bronchial needle aspiration in diagnosis of melioidosis - case series and review of literature
}

\author{
Mujeeb Rahman K.K ${ }^{1}$, Sourin Bhuniya ${ }^{1}$, Bijayini Behera ${ }^{2}$, Prasanta Raghab Mohapatra ${ }^{1}$, \\ Rajesh Kumar ${ }^{3}$, Anjuna Radhakrishnan² \\ ${ }^{1}$ Department of Pulmonary Medicine and Critical Care; ${ }^{2}$ Department of Microbiology; ${ }^{3}$ Department of General \\ Medicine, All India Institute of Medical Sciences, Bhubaneswar, India
}

\begin{abstract}
Endobronchial ultrasound guided transbronchial needle aspiration (EBUS-TBNA) is routinely performed for diagnostic evaluation of mediastinal lymphadenopathy due to various etiologies with excellent sensitivity and specificity. Melioidosis can have atypical features like isolated mediastinal lymphadenopathy mim-
\end{abstract}

Correspondence: Sourin Bhuniya, Room no. 146, OPD block (1st floor), Department of Pulmonary Medicine and Critical Care, All India Institute of Medical sciences, Bhubaneswar 751019, India. Tel. +91.-9438884284.

E-mail: sbhuniya@hotmail.com

Key words: Bronchoscopy; melioidosis; mediastinal lymphadenopathy; endobronchial ultrasound.

Conflict of interest: The authors declare that they have no competing interests, and all authors confirm accuracy.

Contributions: All the authors made a substantive intellectual contribution, performed part of the experiments. All the authors have read and approved the final version of the manuscript and agreed to be accountable for all aspects of the work.

Availability of data and materials: All data generated or analyzed during this study are included in this published article.

Ethics approval and consent to participate: No ethical committee approval was required for this case report by the Department, because this article does not contain any studies with human participants or animals.

Consent for publication: The patients gave their consent to use their personal data for the publication of these case reports and any accompanying images.

Received for publication: 29 December 2019.

Accepted for publication: 18 May 2020.

${ }^{\circ}$ Copyright: the Author(s), 2020

Licensee PAGEPress, Italy

Monaldi Archives for Chest Disease 2020; 90:1221

doi: 10.4081/monaldi.2020.1221

This article is distributed under the terms of the Creative Commons Attribution Noncommercial License (by-nc 4.0) which permits any noncommercial use, distribution, and reproduction in any medium, provided the original author(s) and source are credited. icking as tuberculosis or lymphoma. Differentiation of such atypical melioidosis presentation become difficult due to similar clinical, radiological and even similar EBUS lymph node characteristics. Role of EBUS-TBNA in diagnosing melioidosis is under investigated and sparsely reported. We describe two cases of melioidosis diagnosed by point of care rapid lateral flow immunoassay antigen testing and culture of EBUS-TBNA samples from necrotic mediastinal lymph nodes.

\section{Introduction}

Endobronchial ultrasound-guided transbronchial needle aspiration (EBUS-TBNA) is a minimally invasive procedure that has now replaced mediastinoscopy in sampling mediastinal lymph nodes [1]. The samples are usually sufficient for tissue diagnosis and even for molecular testing with an overall sensitivity of $93 \%$ and specificity $100 \%[2,3]$. Role of EBUS in diagnosis of granulomatous lymphadenitis like tuberculosis and sarcoidosis is well established in the literature [4]. Melioidosis caused by Burkholderia pseudomallei, is difficult to differentiate clinically from tuberculosis as both can present with mediastinal lymphadenopathy with identical imaging and ultrasound features. There is sparse literature on use of EBUS-TBNA in diagnosing melioidosis. Burkholderia pseudomallei infection presenting with isolated mediastinal necrotic lymph nodes is not common and there has been no case series published on the utility of EBUSTBNA and rapid antigen testing in early diagnosing meliodosis. Here we discuss two cases of melioidosis presenting as mediastinal lymphadenopathy that could be diagnosed early by EBUSTBNA and lateral flow antigen testing, with a brief review from available literature.

\section{Case \#1}

A 66-year male with uncontrolled diabetes and hypertension presented with history of fever, generalized weakness and decreased appetite for last three weeks. He was treated with intravenous piperacillin-tazobactam at a private hospital without any improvement, and was referred to our hospital for further evaluation. On general examination, patient was febrile (temp $102^{\circ} \mathrm{F}$ ) and confused but his vital parameters were within normal limits. His respiratory system and neurological examination was unremarkable. There were no signs of meningitis. Laboratory investigations showed neutrophilic leukocytosis with a total count of 
11200/cm, 94\% neutrophils and normal platelet count. His fasting blood sugar was $224 \mathrm{mg} / \mathrm{dL}$ and glycosylated haemoglobin (HbA1C) 11.2\% suggestive of uncontrolled diabetes mellitus. Blood urea level was $61 \mathrm{mg} / \mathrm{dl}$ and creatinine $2.2 \mathrm{mg} / \mathrm{dL}$. Urine showed $2+$ proteinuria suggestive of diabetic nephropathy. Liver function test was within normal limit.

We investigated for the possible causes of fever by laboratory investigations like blood culture, urine culture and serological tests for dengue NS1Ag and Ig M antibody, Malaria antigen, Scrub typhus Ig M and sputum examination for tuberculosis, but all were negative. Computed tomography (CT) of chest showed bilateral normal lung parenchyma with mediastinal lymphadenopathy (Figure 1) Ultrasonography (USG) of abdomen, CT brain, and echocardiography did not reveal any abnormality. The patient was posted for bronchoscopy \& EBUS-TBNA for the evaluation of necrotic mediastinal lymph nodes. Bronchoscopy did not reveal any mucosal or endobronchial lesion and all the segments were visualized normally. EBUS was done using PENTAX EB-1970UK linear EBUS bronchoscope under conscious sedation and showed well defined, oval, heterogenous mediastinal lymph nodes with coagulation necrosis, without any central hilar structure or intranodal vessels over right lower paratracheal (4R), subcarinal (7), and right interlobar $(11 \mathrm{R})$ of sizes $28 \times 45 \mathrm{~mm}, 37 \times 23.1 \mathrm{~mm}, 10.5 \times 21.9$ mm respectively (Figure 1). EBUS guided TBNA was done using 22G EBUS needle (COOK MEDICAL, ECHO -HD-22 EBUS-P) with 3 passes from $4 \mathrm{R}$ and another 3 from 11R. Necrotic material was aspirated and sent for cytology, cell block, and bacterial, fungal culture and Xpert MTB for Mycobacterium tuberculosis. TBNA cytology showed inflammatory exudate without any granuloma or atypical cells. No acid-fast bacilli (AFB) was seen in Ziehl-Neelsen staining, $\mathrm{KOH}$ mount was negative for fungal elements and Xpert MTB was negative for M. tuberculosis. EBUSTBNA sample was positive for capsular polysaccharide antigen of Burkholderia pseudomallei by Active Melioidosis Detect-Lateral Flow Immunoassay test (AMD-LFI, InBios International Inc., USA) and negative for culture. Patient was started on modified renal dose of intra-venous (IV) Meropenem $500 \mathrm{mg}$ q 8 hourly and oral Doxycycline $100 \mathrm{mg} 12$ hourly on the same day of EBUSTBNA based on rapid lateral flow immunoassay report. Blood culture report was available only by next day of EBUS-TBNA, which revealed growth of $B$. pseudomallei, that was sensitive to amoxicillin and clavulanic acid, meropenem, doxycycline, ceftazidime and trimethoprim/sulfamethoxazole. Patient successfully completed 15 days of intensive phase treatment with meropenem and

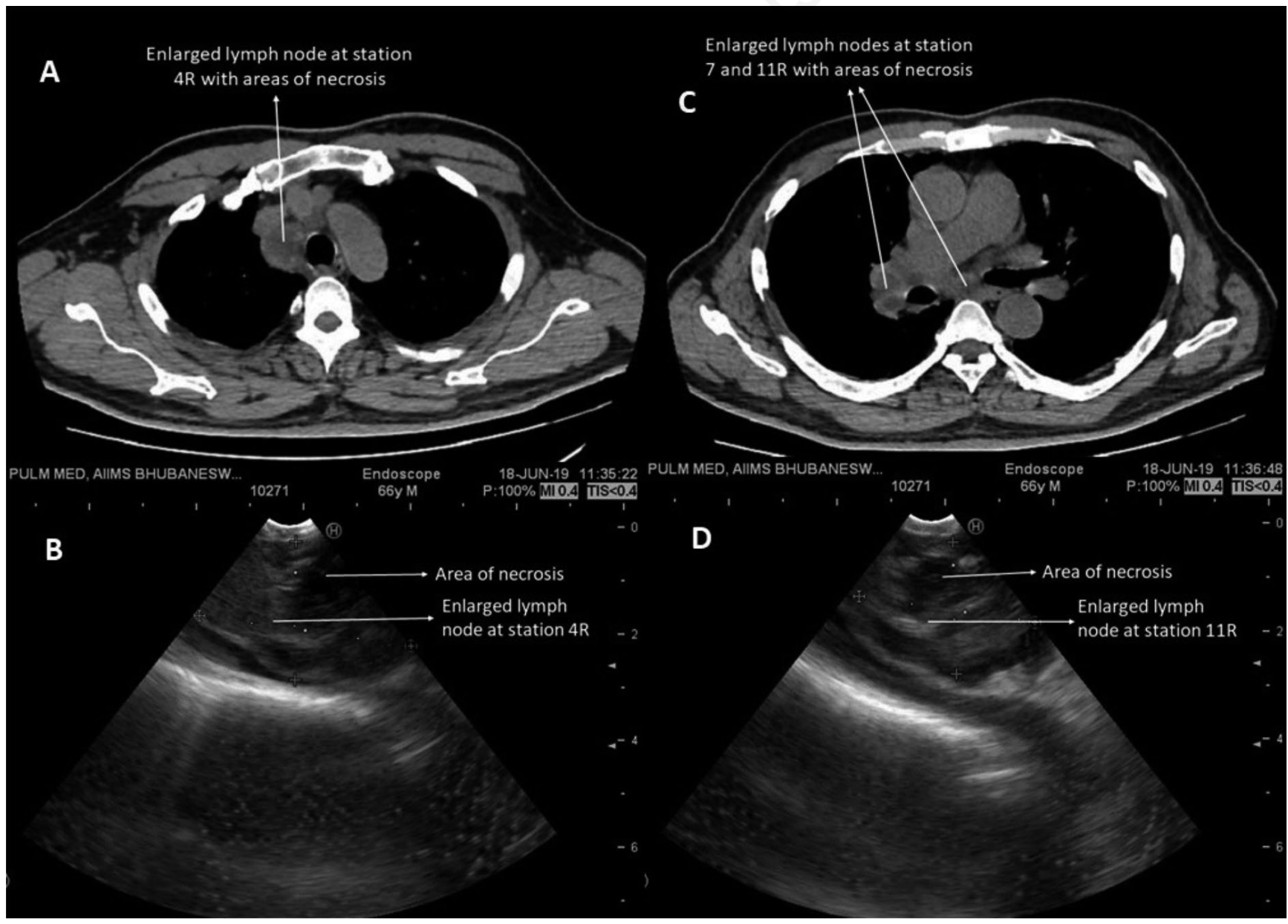

Figure 1. CT scan of thorax showing enlarged mediastinal lymph nodes at station 4R (A), 7 and $11 \mathrm{R}(\mathrm{C})$ with areas of necrosis as shown by arrows. EBUS images showing heterogenous echotexture and coagulation necrosis in station $4 R(B)$ and $11 R(D)$ nodes of the same patient. 
doxycycline with significant clinical improvement and was discharged on oral trimethoprim/sulfamethoxazole (160/800 mg), 2 tablets q 12 hourly for six months. Patient is still on regular monthly follow up and is doing well after 4 months.

\section{Case \#2}

A 74-year male physician, who was diabetic, hypertensive and nonsmoker, presented to us with low persistent fever, loss of appetite and burning micturition for one month. General examination and hemodynamic parameters were normal. Respiratory, cardiac, neurological and abdominal examinations were also unremarkable. Patient was evaluated for fever of unknown origin with complete blood counts, kidney and liver function test, peripheral blood smear, dengue, and scrub typhus serology, malaria antigen test and chest radiograph. These investigations did not reveal any abnormality except mild neutrophilic leukocytosis. Blood culture also did not grow any organism. Chest radiograph was apparently normal but CT thorax showed bilateral normal lung parenchyma with mediastinal lymphadenopathy, sub carinal lymph node of size $30 \times 24 \mathrm{~mm}$ being the largest (Figure 2).

Patient was planned for bronchoscopy and EBUS evaluation of para tracheal and sub carinal lymphadenopathy. Bronchoscopy did not reveal any abnormal endobronchial or mucosal findings. Convex probe EBUS (PENTAX EB-1970UK) showed sub-carinal lymph nodes (station 7) of size $29 \times 19,16.8 \times 21.5,16 \times 12.5 \mathrm{~mm}$, and right lower paratracheal lymph node (station $4 \mathrm{R}$ ) of size $16.7 \times 17.5 \mathrm{~mm}$. Both station lymph nodes were having oval shape, well defined margins and heterogeneous echogenicity with coagulation necrosis sign but without intra nodal vessels or central hilar structures. EBUS-TBNA was done using 22G EBUS needle (COOK MEDICAL, ECHO -HD-22 EBUS-P) form the largest sub carinal lymph node (Figure 2), necrotic pus like material was aspirated in all the 3 passes and sent for cytology, staining for acid fast bacilli, cell block, Xpert MTB, bacterial culture, fungal stain- ing and fungal culture. Smear and Xpert MTB were negative for tuberculosis, but Active Melioidosis Detect- Lateral Flow Immunoassay test (AMD-LFI) was positive and after 5 days the culture of the EBUS-TBNA aspirate yielded B. pseudomallei (Figure 3) that was sensitive to all antimicrobials as mentioned in Case \#1. Patient was started on IV meropenem 1 gm q8 hourly on the same day of EBUS-TBNA based on lateral flow immunoassay report. He completed 2 weeks of intensive phase therapy with significant clinical improvement. He was started on oral maintenance therapy of trimethoprim/sulfamethoxazole (160/800 mg), 2 tablets q12 hourly and is doing well without any complains after 4 months of follow-up.

\section{Discussion}

Melioidosis is a condition due to infection with the gram-negative saprophytic bacteria, $B$. pseudomalle $i$ with high case fatality rate (10-50\%). Mostly endemic to Australia and Thailand, the disease has shown its resurgence in southern and eastern India as well [5-7].

Actual incidence of melioidosis in India is not clear due to lack of standardized national surveillance mechanism, although recent mathematical prediction modelling suggests India bearing a high burden of melioidosis, with this institute alone treating over 100 cases during last 5 years [8-10]. Acute pulmonary melioidosis can present as pneumonia, sepsis and acute respiratory distress syndrome and it commonly occurs in endemic areas. However, patients presenting with subacute or chronic pulmonary symptoms can cause diagnostic challenges for clinicians and mostly present as fever of unknown origin $[11,12]$. In subacute and chronic pulmonary infection, the presentation can mimic chronic infections such as tuberculosis and fungus or malignancy, with radiological findings like necrotic mediastinal lymph nodes, space occupying pulmonary opacities and cavitory lesions. Chronic pulmonary melioidosis can demonstrate nodular, cavitary or fibrotic lesions on chest radiographs [12].

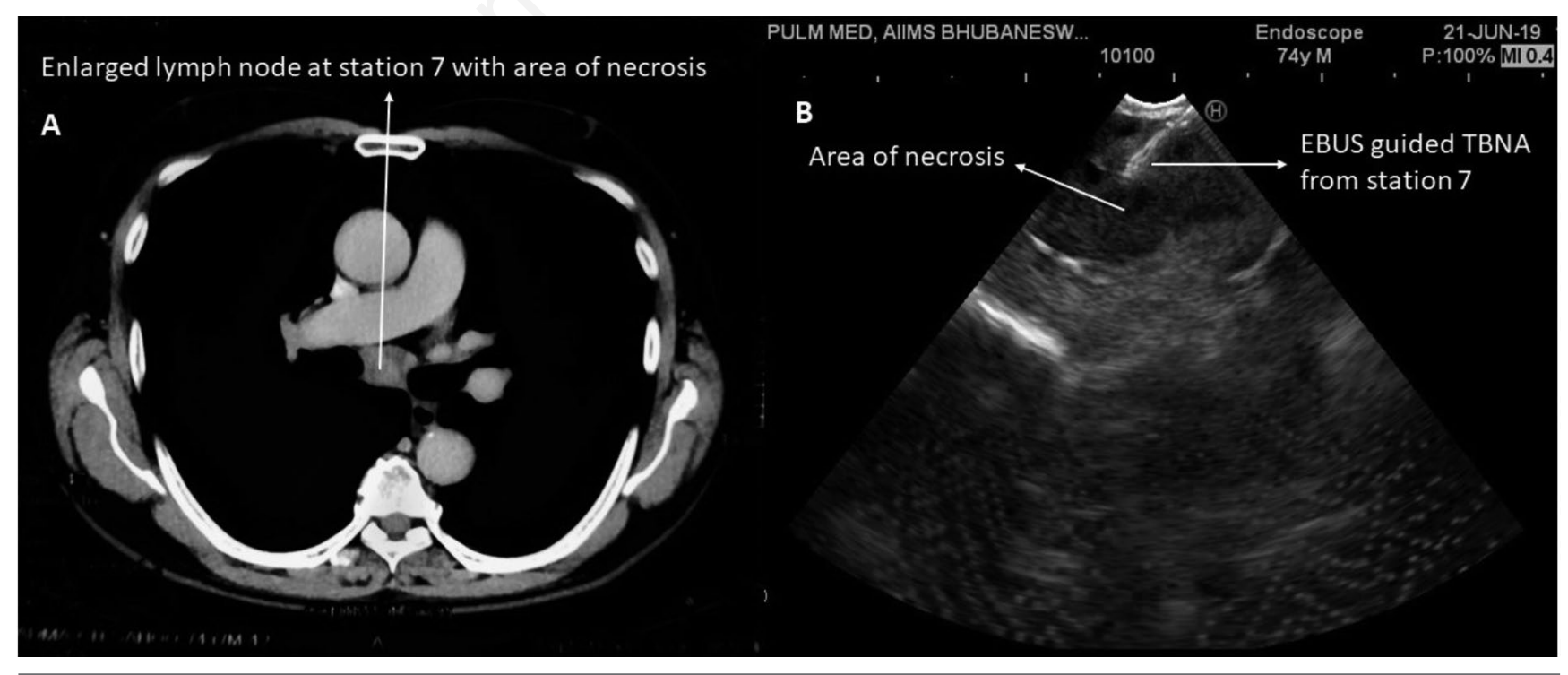

Figure 2. A) CT thorax image of enlarged sub-carinal lymph node (station 7) with area of necrosis. B) EBUS image of the same node with the TBNA needle in-situ as shown by the arrow. 
Culture, though highly specific, has maximum sensitivity up to $60 \%$. Serologic testing like indirect hemagglutination assays can assist but has low sensitivity early in infection and background seropositivity in endemic regions makes positive serology not specific for active disease [13]. Diagnosis is difficult due to inadequacy of conventional bacterial culture identification methods. But antigen detection tests of body fluids using Melioidosis detect- lateral flow assay has been shown to establish diagnosis quickly with sensitivity of $85.7 \%$ and specificity $93.6 \%[14,15]$. Use of enrichment culture and PCR technique is adjunct to conventional culture but the lack of infrastructure and high cost make their use very limited in developing countries like India.

The absence of definite pulmonary parenchymal pathology and presence of isolated necrotic mediastinal lymph nodes had made the diagnosis difficult in our cases. Being a country with high burden of tuberculosis, in cases of fever of unknown origin with necrotic mediastinal lymph nodes first probable diagnosis is usually tuberculosis. Contrast enhanced CT thorax and EBUS are the modalities of choice in such a situation. But the radiological and endobronchial ultrasound features of mediastinal lymph nodes in both tuberculosis and melioidosis are almost similar. Blood culture along with bronchoscopy with bronchoalveolar lavage may be insufficient if there is normal lung parenchyma, but EBUS-TBNA aspirated necrotic material is an excellent method for both $B$. pseudomallei antigen test and culture. There are certain endobronchial ultrasonic features which predict diagnosis of specific entity like benign or malignant lesion, sarcoidosis and tuberculosis. But presence or absence of such feature does not preclude the need of EBUS-TBNA sample cytology, histology and microbiological analysis. For example, compared to sarcoidosis, presence of heterogeneous ultrasound texture and signs of coagulation necrosis are fairly specific for tuberculosis. In comparison to malignant lymph nodes, presence of well-defined margin, small size, triangular shape, presence of central hilar structure, and nodal conglomeration are predictive of benign lymph nodes [13]. But in both of our melioidosis cases bronchoscopy was normal and lymph nodes in endobronchial ultrasound were having similar heterogeneous echo texture, with signs of coagulation necrosis similar to that of tuberculosis.

To the best of our knowledge there is only one case report till date, showing role of linear EBUS-TBNA in diagnosis of melioidosis [16]. Secondly in the literature a case of chronic melioidosis presenting as peripheral lung mass and mimicking malignancy got diagnosed using radial EBUS with sheath technique $[16,17]$. In our first case, positive blood culture report was not available till the time of EBUS-TBNA and mediastinal lymph node aspirate sample was positive for rapid antigen test but negative for culture. In the second case, even though initial blood culture report was negative EBUSTBNA aspirate antigen test and culture was positive. Thus, in both the cases, rapid lateral flow immunoassay test from EBUS-TBNA samples helped us to establish the diagnosis on the same day of the procedure as a 'point of care' testing. Therefore, few days of delay due to waiting for culture report was saved and further deterioration was averted. In the second case, blood culture was sterile and EBUSTBNA sample was the only mode of diagnosis.

In our series, convex probe EBUS-TBNA and lateral flow antigen testing had contributed in establishing early diagnosis of Melioidosis. Ours is probably the only case series in the literature demonstrating the utility of EBUS-TBNA combined with lateral flow antigen testing in the early diagnosis of melioidosis. There should be clinical suspicion of melioidosis in all patients presenting with chronic fever and mediastinal lymphadenopathy in this part of the tropics and they should undergo appropriate investigations to rule out $B$. pseudomallei, especially in those with uncontrolled diabetes mellitus, because the treatment regimen and prognosis markedly differ from that of the usual pathogens.

\section{A Control line

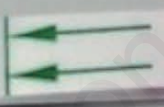 \\ 1500 \\ Sample pad \\ Test line (positive)}
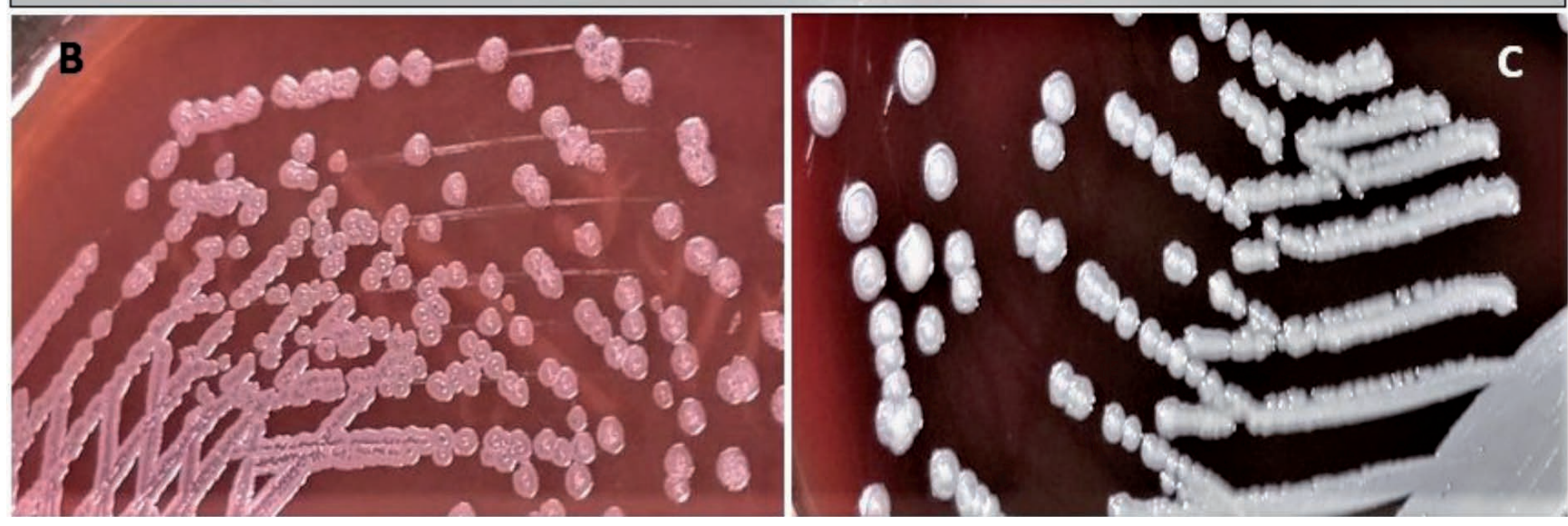

Figure 3. A) Lateral flow immunoassay strip with positive test result. Colonies of Burkholderia pseudomallei grown in Mac Conkey agar (B) and blood agar (C) from the EBUS-TBNA sample. 


\section{References}

1. Navani N, Lawrence DR, Kolvekar S, et al. Endobronchial ultrasound-guided transbronchial needle aspiration prevents mediastinoscopies in the diagnosis of isolated mediastinal lymphadenopathy: a prospective trial. Am J Respir Crit Care Med 2012;186:255-60.

2. Gu P, Zhao Y-Z, Jiang L-Y, et al. Endobronchial ultrasoundguided transbronchial needle aspiration for staging of lung cancer: a systematic review and meta-analysis. Eur J Cancer 2009;45:1389-96.

3. Casadio C, Guarize J, Donghi S, et al. Molecular testing for targeted therapy in advanced non-small cell lung cancer: Suitability of endobronchial ultrasound transbronchial needle aspiration. Am J Clin Pathol 2015;144:629-34.

4. Li W, Zhang T, Chen Y, et al. Diagnostic value of convex probe endobronchial ultrasound-guided transbronchial needle aspiration in mediastinal tuberculous lymphadenitis: a systematic review and meta-analysis. Med Sci Monit 2015;21: 2064-72.

5. Mukhopadhyay C, Chawla K, Krishna S, et al. Emergence of Burkholderia pseudomallei and pandrug-resistant non-fermenters from southern Karnataka, India. Trans R Soc Trop Med Hyg 2008;102:S12-7.

6. Vidyalakshmi K, Lipika S, Vishal S, et al. Emerging clinicoepidemiological trends in melioidosis: analysis of 95 cases from western coastal India. Int J Infect Dis 2012;16:e491-7.

7. Tellapragada C, Shaw T, D'Souza A, et al. Improved detection of Burkholderia pseudomallei from non-blood clinical specimens using enrichment culture and PCR: Narrowing diagnos- tic gap in resource constrained settings. Trop Med Int Health 2017;22:866-70.

8. Limmathurotsakul D, Golding N, Dance DA, et al. Predicted global distribution of Burkholderia pseudomallei and burden of melioidosis. Nat Microbiol 2016;11;1:15008.

9. Currie BJ. Melioidosis: evolving concepts in epidemiology, pathogenesis and treatment. Semin Respir Crit Care Med 2015;36:111-25.

10. Mohapatra PR, Behera B, Mohanty S, et al. Melioidosis. Lancet Infect Dis 2019;19:1056-7.

11. Meumann EM, Cheng AC, Ward L, Currie BJ. Clinical features and epidemiology of melioidosis pneumonia: results from a 21-year study and review of the literature. Clin Infect Dis 2012;54:362-9.

12. Chong VF, Fan YF. The radiology of melioidosis. Australas Radiol 1996;40:244-9.

13. O'Brien M, Freeman K, Lum G, et al. Further evaluation of a rapid diagnostic test for melioidosis in an area of endemicity. $\mathrm{J}$ Clin Microbiol 2004;42:2239-40.

14. Houghton RL, Reed DE, Hubbard MA, et al. Development of a prototype lateral flow immunoassay (LFI) for the rapid diagnosis of melioidosis. PLoS Negl Trop Dis 2014;8:e2727.

15. Fujiwara T, Yasufuku K, Nakajima T, et al. The utility of sonographic features during endobronchial ultrasound-guided transbronchial needle aspiration for lymph node staging in patients with lung cancer. Chest 2010;138:641-7.

16. Zaw KK, Wasgewatta SL, Kwong KK, et al. Chronic pulmonary melioidosis masquerading as lung malignancy diagnosed by EBUS guided sheath technique. Respir Med Case Rep 2019;28:100894.

17. Chan HP, Yip HS. Mediastinal lymphadenopathy: melioidosis mimicking tuberculosis. Trop Med Health 2015;43:93-4. 